\title{
Risk Factors Associated with Leishmaniasis in the Most Affected Provinces by Leishmania infantum in Morocco
}

\author{
Maryam Hakkour (D), 1,2,3 Asmae Hmamouch ${ }^{2},{ }^{2,4}$ Mohamed Mahmoud El Alem (D), 1,2 \\ Abdelhakim Bouyahya $\left(\mathbb{1},{ }^{5}\right.$ Abdelaali Balahbib $\left(\mathbb{0},{ }^{1,2}\right.$ Abdelhak EL Khazraji, ${ }^{6}$ \\ Hajiba Fellah $\left(\mathbb{D}^{1,}, 2\right.$ Abderrahim Sadak $\left(\mathbb{0},{ }^{1}\right.$ and Faiza Sebti ${ }^{1}{ }^{2}$ \\ ${ }^{1}$ Laboratory of Zoology and General Biology, Faculty of Sciences, Mohammed V University in Rabat, Rabat, Morocco \\ ${ }^{2}$ National Reference Laboratory of Leishmaniasis, National Institute of Hygiene, Rabat, Morocco \\ ${ }^{3}$ Agronomy and Veterinary Institute Hassan II, Department of Parasitology, Rabat, Morocco \\ ${ }^{4}$ Laboratory of Microbial Biotechnology, Sciences and Techniques Faculty, Sidi Mohammed Ben Abdellah University, \\ $\mathrm{Fez}$, Morocco \\ ${ }^{5}$ Laboratory of Human Pathologies Biology, Faculty of Sciences, University Mohammed V, Rabat, Morocco \\ ${ }^{6}$ Medical Biotechnology Laboratory, Faculty of Medicine and Pharmacy of Rabat, University Mohammed V, Rabat, Morocco
}

Correspondence should be addressed to Maryam Hakkour; maryam.hakkour@gmail.com

Received 5 February 2020; Revised 17 May 2020; Accepted 1 June 2020; Published 25 June 2020

Academic Editor: Subhada Prasad Pani

Copyright (c) 2020 Maryam Hakkour et al. This is an open access article distributed under the Creative Commons Attribution License, which permits unrestricted use, distribution, and reproduction in any medium, provided the original work is properly cited.

\begin{abstract}
Background. Human leishmaniasis, both visceral and cutaneous, has been reported in Morocco for centuries and constitutes a serious public health problem. However, the evolution of this pathology depends on several factors such as ecological, socioeconomic, and climatic conditions. The risk study of the affected foci is of great value for the control and surveillance of this endemic disease, especially in the provinces where Leishmania infantum predominates. Methods. This study concerned nine provinces located in the extreme and central north of Morocco (Taounate, Taza, Chefchaouen, Al Hoceima, Larache, Tétouane, Tanger-Assilah, M'diq-Fnideq, and Fahs-Anjra Provinces). In this work, leishmaniasis cases (VL and CL) were subjected to an epidemiological study which was performed using a linear regression model to identify the impact as well as the interaction between all predictor variables on the distribution of leishmaniasis in this region. Results. During the period 1997-2018, a total of 6 128 cases of VL and CL were recorded in the study area. Our results showed that among demographic factors studied, urbanization showed significance for both cutaneous and visceral forms $(P<0.05)$. Regarding the environmental factors, the humidity and the altitude were significant for both CL and VL $(P<0.05)$, while the temperature and the normalized difference vegetation index (NDVI) showed a significance only for VL. Moreover, trends in season of occurrence revealed that wet season (October to April) had a higher incidence of leishmaniasis compared to the dry season (May to September) specifically for CL. As for socioeconomic factors, poverty was the only factor that influences the spread of VL. Finally, the distance from endemic foci showed significance for both VL and LC $(P<0.05)$. Conclusion. Our study revealed that the risk factor associated with cutaneous and visceral leishmaniasis in northern Morocco could help in the establishment of a prediction program.
\end{abstract}

\section{Introduction}

Environmental conditions and socioeconomic and demographic factors have a serious impact on human health, particularly on vector-borne infectious diseases including leishmaniasis [1]. In fact, leishmaniasis is an endemic parasitic disease in most tropical regions of the world with approximately two million new human cases reported each year [2]. In Morocco, it is considered among the main endemic disease [3]. The parasite species belonging to the genus Leishmania are responsible for different clinical pathologies (visceral and the cutaneous form) according to the localization of these parasites in mammalian tissues [4]. 
The visceral leishmaniasis (VL) caused by Leishmania infantum has been endemic in Morocco for several decades. This disease was essentially limited in the north of Morocco but has shown a remarkable extension over time, and many cases have been observed in other regions [5]. Between 1990 and 2014, the average annual incidence rate was $0.4 \mathrm{VL}$ cases per 100000 inhabitants [6]. Concerning the cutaneous leishmaniasis (CL), it is caused by three Leishmania species: Leishmania major, Leishmania tropica, and Leishmania infantum with more than 3700 cases reported yearly in this country between 1997 and 2018. The peaks are registered in 2010 and 2018 with, respectively, 8707 and 9700 cases [7].

In Morocco, the epidemiological situation of leishmaniasis as well as the distribution of Leishmania species varies since the country comprises several geographical areas with different socioeconomic, environmental, and ecological characteristics [8]. In this context, the purpose of this study was to establish epidemiological data on leishmaniasis in the northern region of Morocco known to be moderately affected by CL and highly affected by the VL during the 21year period (1997-2018) and to determine the impact of environmental fluctuations (temperature, rainfall, humidity, altitude, and NDVI) and the role of demographic factors (population density), socioeconomic (poverty and vulnerability rate), and other indicators (distance to leishmaniasis foci) as risk factors on the propagation of the leishmaniasis in this region.

The epidemiological situation of leishmaniases in the studied provinces was statistically supported in order to identify the various factors responsible for the evolution of this pathology: demographic factors (population density, urbanization, age, and sex); environmental factors (temperature, rainfall, humidity, altitude, NDVI, and seasonality of infection); socioeconomic factors (poverty rate and vulnerability rate), and other factors (distance to leishmaniasis foci). A linear regression model was chosen to study the impact of each factor on all the provinces as well as on each province to illustrate the different modeling possibilities.

\section{Methods}

2.1. Study Area. This study was established in nine provinces located in the extreme north and central north of Morocco: Taounate, Taza, Chefchaouen, Al Hoceima, Larache, Tetouan, Tanger-Assilah, M'diq-Fnideq, and Fahs-Anjra Provinces (Figure 1).

These zones are part of the north, northwest, and northeast section of the Arc du Rif; they are also known as "Jbala". Located in the North of Morocco ( $35^{\circ} 15^{\prime} 00^{\prime \prime}$ North, $5^{\circ} 56^{\prime} 00^{\prime \prime}$ West), they are bordered to the north by the Strait of Gibraltar and the Mediterranean Sea, to the west by the Atlantic Ocean, to the southwest by the region of Rabat-SaleKenitra, in the south by the provinces of Sefrou and Moulay Yaacoub, and in the east by the region of the Oriental [8].

On the climatic level, these provinces are characterized by a Mediterranean climate with cold and wet winter. The total population in this study area is approximately 4446757 according to the 2014 General Population and Housing Census (RGPH) [9]. Regarding the urbanization rate, it varies between $12.5 \%$ recorded in Chefchaouen Province and $100 \%$ recorded in Fahs-Anjra Province (Table 1).

2.2. Data Collection. A total of 6128 cases were registered in these provinces of study between 1997 and 2018. Data on human cases were obtained from the Epidemiology and Disease Control Directorate [5]. Environmental variables (temperature, humidity, altitude, and NDVI) were obtained from https://fr.climate-data.org, while normalized difference vegetation index data were collected from the Royal Center for Remote Sensing Space (http://www.crts.gov.ma/ bulletins-campagne-agricole).

Data on poverty and the vulnerability rate were obtained from the High Commission for Planning, indicators of poverty and vulnerability in Morocco [10]. However, data on the urbanization rate and population were obtained from the monograph of the Tangier-Tetouan-Al Hoceima and FezMeknes regions [11].

According to the High Commission for Planning, the poverty rate is the proportion of individuals (or households) with a low standard of living. Indeed, it is estimated that in 2007, people earning less than 3834 MAD (380€) in urban areas and 3569 MAD (350€) in rural areas in Morocco are considered poor [10]. However, the vulnerability rate to poverty is the proportion of individuals who spent annually 1.5 times more than the threshold of poverty rate. Regarding the urbanization rate, it is the ratio of the urban population to the total population [12].

2.3. Statistical Analysis. Statistical analysis was performed using software R version 3.3.3 (http://www.R-project.org). The regression model was applied to evaluate the impact of several factors. The correlation between the total number of leishmaniasis cases and socioeconomic, demographic, and environmental predictors and distance with leishmaniasis foci (in $\mathrm{km}$ ) was tested using Pearson rank correlation as previously described [13]. The significance level was $P<0.05$.

\section{Results}

3.1. Temporal Distribution of VL and CL Cases between 1997 and 2018 in the Study Area. According to the Moroccan Ministry of Health, a total of 6128 cases of VL and CL were recorded in the study area. These provinces are known as moderately affected by the CL and strongly affected by the VL compared to the other provinces of the kingdom. Cutaneous form represented $6.34 \%$, the majority of cases were recorded in Taounate Province (45.90\%, 2 271/4 947) and Taza Province (39.94\%, 1 976/4 947) followed by Larache, Al Hoceima, and Chefchaouen Provinces which recorded 4.54\% (225/4 947), 4.08\% (202/4 947), and 3.3\% (163/4 947), respectively. Concerning the provinces of Tetouan, TangierAssilah, Fahs-Anjra, and M'diq-Fnideq, they have recorded $1.53 \%$ (76/4 947), 0.34\% (17/4 947), 0.22\% (11/4 947), and $0.12 \%$ (6/4 947) (Figure 2).

Concerning visceral leishmaniasis, it represents $25.33 \%$ of reported cases in Morocco and is distributed as follows: 29.88\% (353/1 181) in Taounate Province, 25.4\% (300/1 181) 


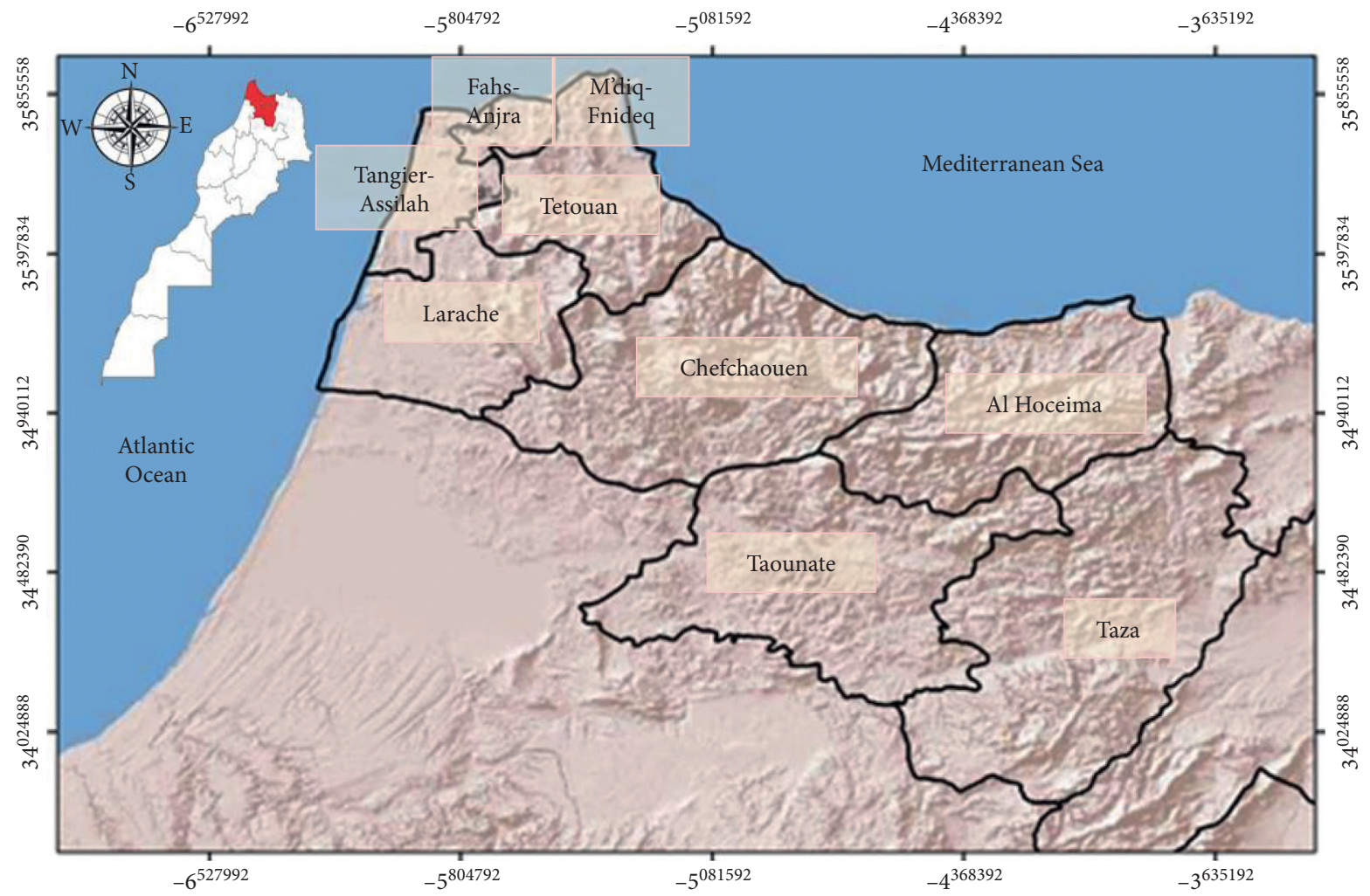

FIgURE 1: Study area.

TABLe 1: Population, urbanization rate, and poverty rate by province of the study [9].

\begin{tabular}{lcccc}
\hline Administrative region & Province & Population & Urbanization rate (\%) & Poverty rate (\%) \\
\hline & Tanger-Assilah & 1065601 & 94.3 & 2.9 \\
& Fahs-Anjra & 76447 & 100.0 & 13.8 \\
& M'diq-Fnideq & 209897 & 94.3 & 3.2 \\
\multirow{2}{*}{ Tanger-Tetouan-Al Hoceima } & Larache & 496687 & 53.5 & 12.2 \\
& Chefchaouen & 457432 & 12.5 & 18.8 \\
& Tetouan & 550374 & 72.3 & 9.7 \\
& Al Hoceima & 399654 & 32.5 & 12.7 \\
\hline \multirow{2}{*}{ Fez-Meknes } & Taounate & 662246 & 13.0 & 23.4 \\
& Taza & 528419 & 39.3 & 15.1 \\
\hline
\end{tabular}

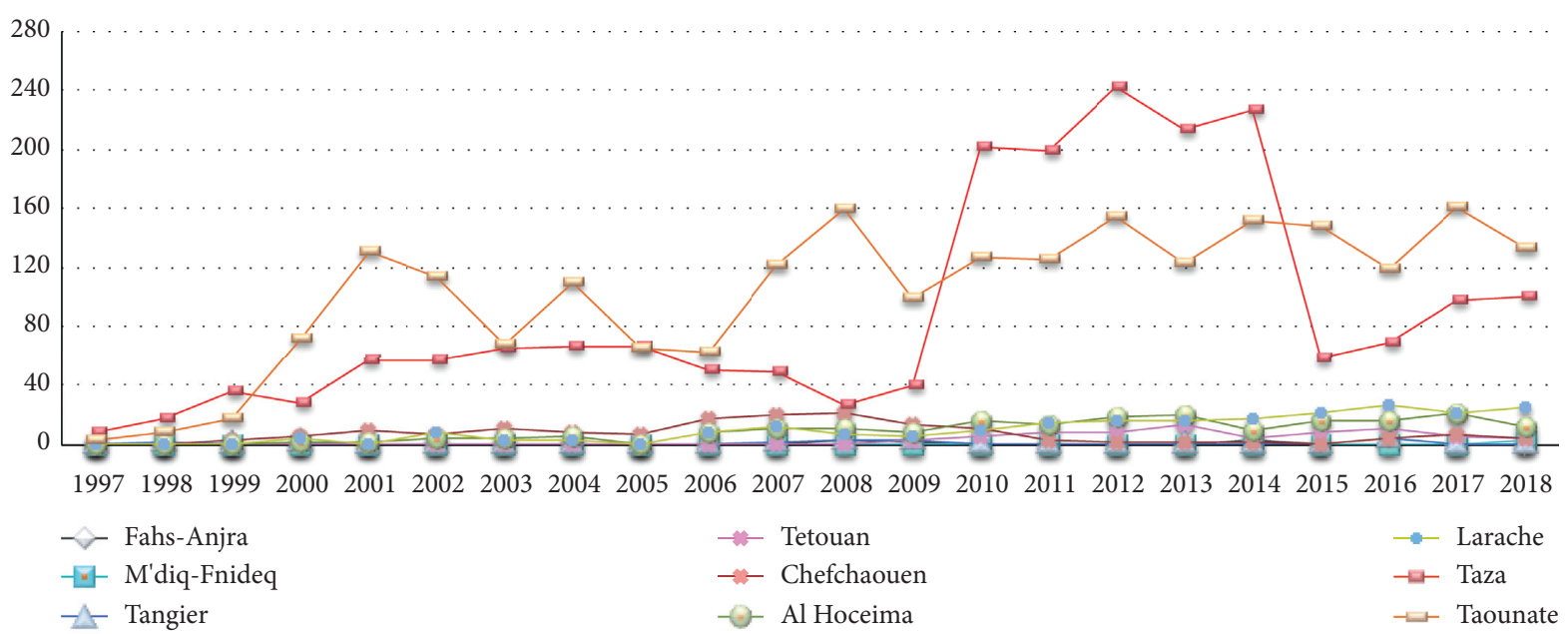

FIgUre 2: Cutaneous leishmaniasis cases between 1997 and 2018 in the study area. 
in Chefchaouen Province, $23.11 \%$ (273/1 181) in Taza Province, $14 \%$ (165/1 181) in Al Hoceima Province, 3.38\% (40/1 181) in Tetouan Province, 3.04\% (36/1 181) in Larache Province, $0.67 \%$ (8/1 181) in M'diq-Fnideq Province, $0.33 \%$ (4/1 181) in Tangier-Assilah Province, and 0.16\% (2/1 181) in Fahs-Anjra Province (Figure 3).

\subsection{Risk Factors Associated with LC and LV}

3.2.1. Demographic Factors. Different demographic variables were studied in order to know which one influences the annual number of cases in each province.

(1) Population Density. Statistical analysis by linear regression (Table 2) showed that the distribution of leishmaniases does not take into account the number of inhabitants $(P$ value $>0.05)$ with a low coefficient of correlation recorded as well as for CL $\left(R^{2}=0.048\right)$ and for VL $\left(R^{2}=0.020\right)$.

The distribution of the population in the nine provinces of study varies between 42914 and 1136967 according to the data from the High Commission for Planning [9]. The statistical analysis between 1997 and 2018 by province showed that the number of inhabitants does not influence the number of cases of the CL and the VL except for the province of Tangier-Assilah where a correlation coefficient was 1 and a significant $P$ val$\mathrm{ue}=0.003$ was detected between the number of cases of VL and the rate of population.

(2) Urbanization. In the study provinces, the rate of urbanization varies from one province to another. The analysis of the results showed that the status of the province in terms of urbanization influences the number of cases of CL and VL. Thus, the number of cases increases with the decrease in the rate of urbanization. Since, in the most affected provinces Taounate (2 271 cases of CL and 353 cases of VL) and Taza (1 976 cases of CL and 273 cases of VL), the rate of urbanization was $13.02 \%$ and $39.36 \%$, respectively. In the moderately affected provinces Al Hoceima (202 cases of CL and 165 cases of VL), Chefchaouen (136 cases of CL and 300 cases of $\mathrm{VL}$ ), and Larache (225 cases of CL and 36 cases of VL), the rate of urbanization was around $12.5 \%, 32.5 \%$, and $53.5 \%$, respectively. In the provinces slightly affected, namely, Tetouan (76 cases of CL and 40 cases of VL), Tangier-Assilah (17 cases of CL and 4 cases of VL), M'diq-Fnideq ( 6 cases of $\mathrm{CL}$ and 8 cases of VL), and Fahs-Anjra (11 cases of CL and 2 cases of $\mathrm{VL}$ ), it is characterized by the rate of urbanization of $72.31 \%, 94.30 \%, 94.30 \%$, and $100 \%$, respectively.

The statistical test gave a correlation coefficient $R^{2}=0.33$ for CL and $R^{2}=0.83$ for VL with a $P$ value $<0.05$. This significant finding revealed that rural provinces were more affected than urban provinces (Table 2).

(3) Age and Sex. The statistical study of the reported cases of leishmaniasis showed that there is no significant difference between the sexes $\left(\chi^{2}=0.58, \mathrm{~d} f=1, P=0.44\right)$, with a slight predominance of the female sex ( $51.08 \%$ women vs. $48.91 \%$ men; sex ratio $\mathrm{F} / \mathrm{M}=1.04$ ) (Figure 4).
Regarding the distribution of affected ages, all age groups are concerned with leishmaniasis. However, children under 10 years were the most affected with $48 \%$ of cases $\left(\chi^{2}=1060.8, \mathrm{~d} f=5, P<2.2 e-16\right)$.

\subsubsection{Environmental Factors}

(1) Temperature. The statistical study, using linear regression, of the impact of temperature between the provinces (Table 3) showed that the distribution of CL does not take into account the rate of temperature; while for VL, a correlation coefficient of $R^{2}=0.48$ was calculated with a $P$ value $=0.03$, so the cases of VL take into account the temperature rate.

However, the analysis of the impact of annual temperature variations on the annual number of cases by province showed that this result was significant only in Al Hoceima $(P$ value $<0.05, R^{2}=0.32$ ) and Larache Provinces ( $P$ value $\left.<0.05, R^{2}=0.38\right)$. On the other hand, the study showed that the number of VL cases takes into consideration the variation of temperature exclusively in Taza Province ( $P$ value $=0.001, R^{2}=0.41$.

(2) Rainfall. Regarding the rate of rainfall, the results of the analysis showed that this factor does not impact the distribution for both CL $\left(P\right.$ value $\left.=0.7, R^{2}=0.02\right)$ and VL $(P$ value $\left.=0.9, R^{2}=0.003\right)$ (Table 3$)$.

(3) Humidity. The analysis of the impact of humidity showed that this factor influences the distribution of leishmaniases with a significant value detected for both CL ( $P$ value $\left.=0.002, R^{2}=0.74\right)$ and $\operatorname{VL}\left(P\right.$ value $\left.=0.001, R^{2}=0.81\right)$ (Table 3).

In fact, the provinces reporting the highest number of cases are characterized by a less humid climate compared with other provinces. However, Taounate and Taza Provinces are classified as bioclimatic zones ranging from subhumid to semiarid with an average humidity of $54.4 \%$ and $58 \%$, respectively. As for the other provinces with fewer cases (Chefchaouen, Al Hoceima, Larache, Tetouan, TangierAssilah, M'diq-Fnideq, and Fahs-Anjra), they are classified in a bioclimatic zone ranging from per-humid to subhumid area with an average humidity of $61 \%, 72.6 \%, 73 \%, 70.3 \%$, $72.2 \%, 70.3 \%$, and $71.2 \%$, respectively, detected in these provinces.

(4) Altitude. The statistical study of the impact of altitude revealed that the number of leishmaniasis cases increases with the increase of the altitude $(P$ value $<0.05)$ with a high coefficient of correlation $\left(R^{2}=0.65\right.$ for CL) and $\left(R^{2}=0.93\right.$ for VL) (Table 3).

(5) NDVI. Regarding the vegetation index (NDVI), the analysis of the results showed that only the number of cases of VL takes into account this factor with a low $P$ value (=0.0003). However, a high $P$ value was calculated for the CL $(P$ value $=0.32)$ which indicates that the latter does not take into account the NDVI (Table 3 ). 


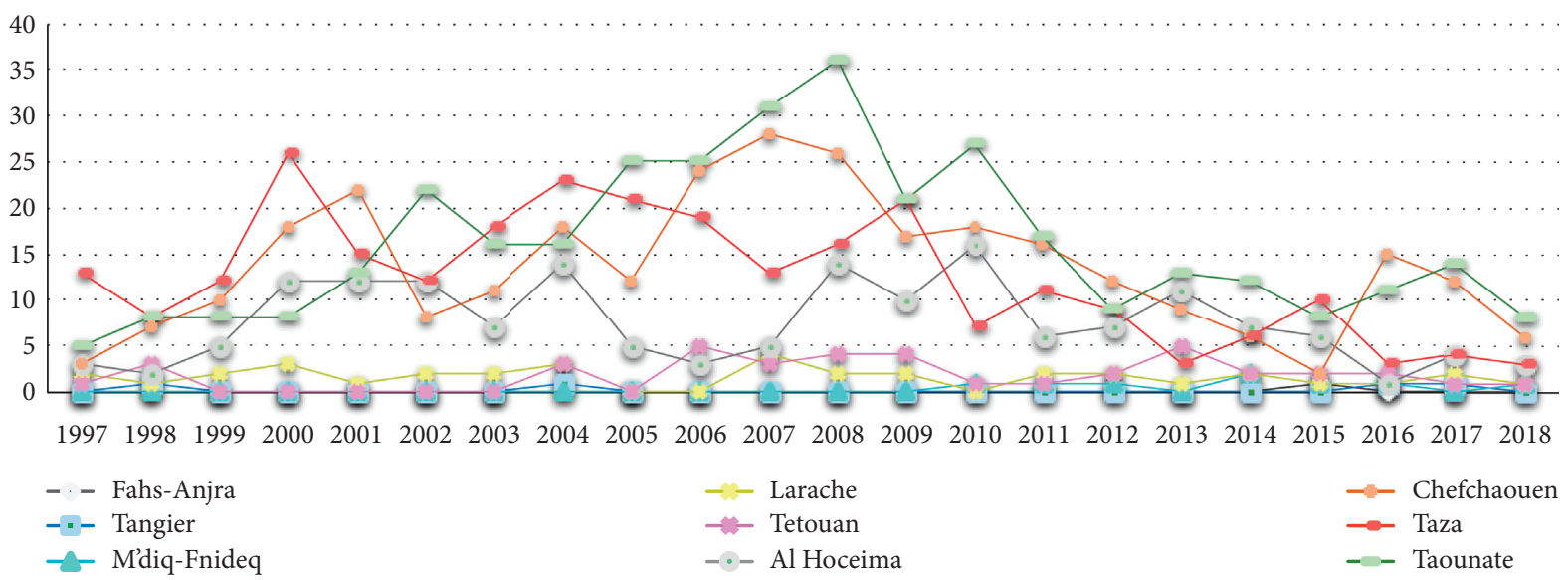

FIGURE 3: Visceral leishmaniasis cases between 1997 and 2018 in the study area.

TABLE 2: Results of the linear regression of leishmaniasis in relation to demographic factors.

\begin{tabular}{lcccccc}
\hline \multirow{2}{*}{ Factor } & \multirow{2}{*}{ Variable } & & \multicolumn{2}{c}{ Coefficient } & \multirow{2}{*}{ T-statistic } & \multirow{2}{*}{$P$ value (significance) } \\
& & & Intercept & Variable & & \\
\hline \multirow{3}{*}{ Demographic } & \multirow{2}{*}{ Population density } & CL & $2.008 e+02$ & $7.074 e-04$ & $F_{(1,7)}=0.3529$ & $0.5712(\mathrm{NS})$ \\
& & VL & $9.573 e+01$ & $7.197 e-05$ & $F_{(1,7)}=0.1395$ & $0.7198(\mathrm{NS})$ \\
& \multirow{2}{*}{ Urbanization } & $\mathrm{CL}$ & 11.000 & 152.000 & $F_{(7,1)}=1.527 e+04$ & $0.006231(\mathrm{~S})$ \\
& & $\mathrm{VL}$ & 345.432 & -3.767 & $F_{(1,7)}=36.140$ & \\
\hline
\end{tabular}

NS = not significant; $\mathrm{S}=$ significant

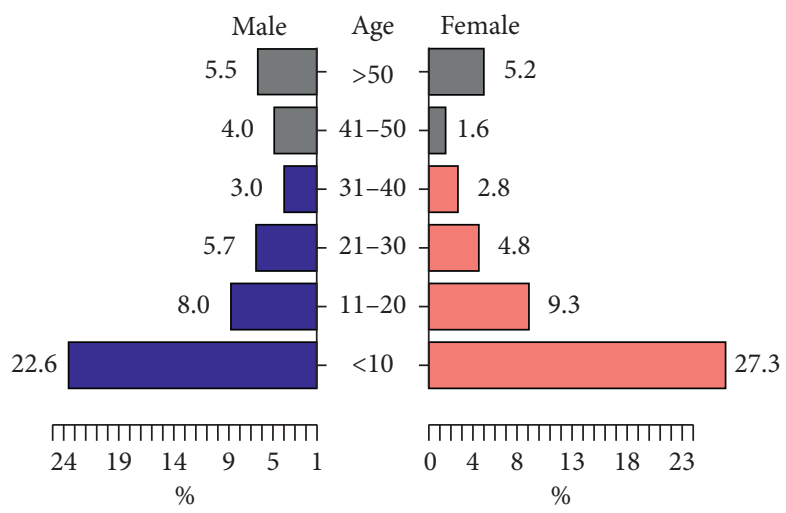

FIgURE 4: Distribution of leishmaniasis according to age and sex.

(6) The Seasonality of Infection. The trends of the season onset revealed that the incidence of leishmaniasis was higher during the wet season (October to April) compared to the dry season (May to September) specifically for $\mathrm{CL}$ $\left(\chi^{2}=55.323 \mathrm{~d} f=1, P\right.$ value $\left.=1.023 e-13\right)$. For VL, a slight dominance of the wet season was noted $\left(\chi^{2}=0.820, \mathrm{~d} f=1, P\right.$ value $=0.36)($ Table 4$)$

\subsubsection{Socioeconomic Factors}

(1) Poverty Rate. In the study area, the poverty rate varies between $2.9 \%$ and $23.4 \%$ according to the data from the High Commission for Planning [9]. The analysis of the results, between provinces, showed that the cumulative number of
CL cases recorded does not depend on poverty ( $P$ value $\left.=0.06, R^{2}=0.41\right)$. However, the recorded cases of $\mathrm{VL}$ takes into account the variation of the poverty rate $(P$ value $=0.005, R^{2}=0.69$ ) .

By studying the impact of this factor on the distribution of leishmaniasis cases in each province over the years (from 2004 to 2018), it was showed that the latter does not affect the distribution of the CL cases where $P$ value was greater than 0.05 .

Nevertheless, this factor affects the number of cases of $\mathrm{VL}$ in four provinces where the number of cases decreased with the decrease in the poverty rate; these are Chefchaouen $\left(P \quad\right.$ value $\left.=0.01, \quad R^{2}=0.40\right)$, Taounate $(P$ value $=0.01$, $\left.R^{2}=0.39\right)$, Taza $\left(P\right.$ value $\left.=0.01 ; R^{2}=0.38\right)$, and M'diq-Fnideq $\left(P\right.$ value $\left.=0.04, R^{2}=0.28\right)$ (Table 5$)$. 
TABLE 3: Results of the linear regression of leishmaniasis in relation to environmental factors.

\begin{tabular}{|c|c|c|c|c|c|c|}
\hline \multirow{2}{*}{ Factor } & \multirow{2}{*}{ Variable } & & \multicolumn{2}{|c|}{ Coefficient } & \multirow{2}{*}{$T$-statistic } & \multirow{2}{*}{$P$ value (significance) } \\
\hline & & & Intercept & Variable & & \\
\hline \multirow{10}{*}{ Environmental } & \multirow{2}{*}{ Temperature } & $\mathrm{CL}$ & 9294,5 & $-493,13$ & $F_{(1,7)}=0.817$ & 0.396 (NS) \\
\hline & & $\mathrm{VL}$ & 3136.10 & -169.44 & $F_{(1,7)}=6.609$ & $0.037(\mathrm{~S})$ \\
\hline & \multirow{2}{*}{ Rainfall } & CL & 1075.417 & -0.8118 & $F_{(1,7)}=0.1614$ & $0.6999(\mathrm{NS})$ \\
\hline & & VL & 161.07396 & -0.04609 & $F_{(1,7)}=0.02006$ & 0.8913 (NS) \\
\hline & \multirow{2}{*}{ Humidity } & $\mathrm{CL}$ & 7764.18 & -107.5 & $F_{(1,7)}=20.83$ & $0.0025(\mathrm{~S})$ \\
\hline & & VL & 1333.9 & -17.92 & $F_{(1,7)}=31.54$ & $0.001(\mathrm{~S})$ \\
\hline & \multirow{2}{*}{ Altitude } & $\mathrm{CL}$ & -62.665 & 2.3068 & $F_{(1,7)}=13.13$ & $0.0084(\mathrm{~S})$ \\
\hline & & VL & 14.342 & 0.4403 & $F_{(1,7)}=100.8$ & $2.083 e-05(\mathrm{~S})$ \\
\hline & \multirow[t]{2}{*}{ NDVI } & CL & 169.00 & -114.40 & $F_{(2,5)}=1.535$ & 0.3202 (NS) \\
\hline & & $\mathrm{VL}$ & 158.00 & -141.60 & $F_{(2,5)}=107.5$ & $1.3(\mathrm{~S})$ \\
\hline
\end{tabular}

NS = not significant; $S=$ significant.

TABLE 4: The onset season of leishmaniasis.

\begin{tabular}{cccc}
\hline & Season & Percentage $(\%)$ & $P$ value \\
\hline \multirow{2}{*}{ CL } & Dry & 38.70 & $1.023 e-13$ \\
& Wet & 61.30 & \\
\multirow{2}{*}{ VL } & Dry & 44.87 & 0.365 \\
\hline
\end{tabular}

(2) Vulnerability Rate. The vulnerability rate varies between $8 \%$ and $23.5 \%$ in the study area. Statistical analysis by linear regression (Table 5) showed that the distribution of leishmaniasis does not depend on this factor neither for CL $(P$ value $\left.=0.06, R^{2}=0.40\right)$ nor for VL $(P$ value $=0.065$, $\left.R^{2}=0.42\right)$.

3.2.4. Other Factors (Distance to Foci of Leishmaniasis). The statistical study of the impact of the neighborhood with known foci of leishmaniasis showed that the number of leishmaniasis cases increases with the decrease of the distance $(P$ value $<0.05)$ with a high coefficient of correlation recorded for both $\mathrm{CL}\left(R^{2}=0.77\right)$ and $\mathrm{VL}\left(R^{2}=0.71\right)$ (Table 6).

\section{Discussion}

This study concerned nine provinces located in the extreme north and central north of Morocco, namely, Taounate, Taza, Chefchaouen, Al Hoceima, Larache, Tetouan, TangerAssilah, M'diq-Fnideq, and Fahs-Anjra Provinces.

According to the Moroccan Ministry of Health, 7.63\% of the leishmaniasis cases reported were recorded in these provinces ( $n=6128 / 80299$ ). In terms of type, the cutaneous form represented $6.34 \%$ of the cases declared $(n=4947 / 78$ $001)$, while the visceral form represented $51.39 \%(n=1181 / 2$ 298).

The results of the retrospective study showed that the majority of leishmaniasis cases were recorded in Taounate and Taza Provinces, followed in the decreasing order by Chefchaouen, Al Hoceima, Larache, Tetouan, TangerAssilah, M'diq-Fnideq, and Fahs-Anjra provinces. Concerning Taounate and Taza Provinces, they are known by their epidemic past with respect to CL and VL [14-16]. The epidemiological profile in these two provinces was widely described by researchers, who have suggested that the high number of cases can be explained by the fact that the parasite could have been present in rural localities and when it was introduced into periurban areas, and it found conditions conducive to an epidemic, including environmental changes associated with rapid and unplanned development, increased density of man and sandfly, and a decrease in immunity of populations [16].

The epidemiological survey carried out in Taounate Province showed that the number of cases has been increasing since 2001 with peaks recorded in 2008, 2012, 2014, and 2017. However, in Taza Province, a sharp increase has been raised since 2010. This increase was mainly explained by the active screening carried out following the implementation of the response action plan which covered the period from 2010 to 2012 . This plan had as objective, on the one hand, the reduction in the incidence of cutaneous leishmaniasis by $50 \%$ at the end of 2012 at the level of the 16 main epidemic foci including Taounate and Taza and on the other hand the taking charge of $100 \%$ of cases of visceral and cutaneous leishmaniasis.

However, in the other provinces of the extreme north, peaks in the number of CL cases were recorded in three provinces (Larache, Al Hoceima, and Chefchaouen). This number is continuously increasing in the provinces of Larache and Al Hoceima, while in the province of Chefchaouen, a remarkable decrease has been noted. In the rest of the provinces studied, the disease is endemic with a few cases declared annually. Concerning the visceral form, a strong declaration of cases has been recorded particularly in Chefchaouen and Al Hoceima Provinces since the beginning of the census of cases in 1997. Several factors may explain the increase in the number of cases in the provinces of the extreme north. On the one hand, the neighborhood of these provinces with several foci of CL and VL, such as Sidi Kacem [17], Ouazzane [17], Taounate [15], and Taza [16]. On the other hand, through the activities of the population and their movement to unscathed areas [18].

In addition to these epidemiological studies, measures to control this pathology must take into account the parasite cycle since each species of the Leishmania genus has its own sandfly species and its own reservoir, and each focus is characterized by the species which circulates as well as the 
TABLE 5: Results of the linear regression of leishmaniasis in relation to socioeconomic factors.

\begin{tabular}{lcccccc}
\hline \multirow{2}{*}{ Factors } & \multirow{2}{*}{ Variable } & & \multicolumn{2}{c}{ Coefficient } & \multirow{2}{*}{-statistic } & \multirow{2}{*}{$P$ value (significance) } \\
& & & Intercept & Variable & & \\
& \multirow{2}{*}{ Poverty rate } & CL & -534.68 & 87.29 & $F_{(1,7)}=4.99$ & $0.060(\mathrm{NS})$ \\
Socioeconomic & VL & -91.537 & 17.932 & $F_{(1,7)}=15.7$ & $0.0054(\mathrm{~S})$ \\
& & $\mathrm{CL}$ & -1007.68 & 99.41 & $F_{(1,7)}=4.777$ & $0.06(\mathrm{NS})$ \\
& Vulnerability rate & VL & -124.235 & 16.306 & $F_{(1,7)}=5.268$ & 0.06 (NS) \\
\hline
\end{tabular}

NS = not significant; $S=$ significant.

TABLE 6: Results of the linear regression of leishmaniasis in relation to factor of distance to foci of leishmaniasis (in $\mathrm{km}$ ).

\begin{tabular}{lcccccc}
\hline \multirow{2}{*}{ Factors } & \multirow{2}{*}{ Variable } & & \multicolumn{2}{c}{ Coefficient } & \multirow{2}{*}{-statistic } & \multirow{2}{*}{$P$ value (significance) } \\
& & & Intercept & Variable & & \\
\hline \multirow{2}{*}{ Other indicators } & \multirow{2}{*}{ Distance to foci of leishmaniasis (in km) } & CL & 1732.68 & -11.510 & $F_{(1,7)}=24.7$ & $0.0016(\mathrm{~S})$ \\
& & VL & 311.90 & -1.758 & $F_{(1,7)}=17.57$ & $0.004(\mathrm{~S})$ \\
\hline
\end{tabular}

$\mathrm{S}=$ significant.

risk factors likely to influence its development. However, molecular investigations recently carried out in these provinces showed the coexistence of $L$. tropica besides $L$. infantum with a predominance of the latter species $[19,20]$.

Regarding the risk factors associated with CL and VL, several elements influence the dynamics and functioning of the Leishmania cycle such as ecological, socioeconomic, and climatic conditions [21]. The risk study of the affected foci is of great use for the control and surveillance of endemic diseases, including leishmaniasis. In our study, the epidemiological situation of leishmaniasis was supported by a statistical analysis in order to identify the various factors such as demographic, environmental, and socioeconomic factors, likely to have a remarkable impact on the evolution of this pathology.

Among the demographic factors studied, the analysis of the results showed that the province's status in terms of urbanization greatly influences the number of CL and VL cases. Indeed, the number of cases increases with the decrease in the urbanization rate. In the most affected provinces (Taounate, Taza, Al Hoceima, and Chefchaouen), the urbanization rate was around $13.02 \%, 39.36 \%, 12.5 \%$, and $32.5 \%$, respectively. In the moderately affected province of Larache, the urbanization rate was around $53.5 \%$. As for the weakly affected provinces: Tetouan, Tanger-Assilah, M'diqFnideq, and Fahs-Anjra, they had an urbanization rate of around $72.31 \%, 94.30 \%, 94.30$, and $100 \%$, respectively.

The statistical results showed and confirmed that urbanization affects the spread of leishmaniasis with the exception of Taza Province. In fact, the majority of Taza cases were recorded in rural areas (Oued Amlil, Bouchefaa, Bouhlou, and Bni Frassen). The urban sector of Taza Haut that has accumulated the totality of cases (614 cases) is characterized by its particular geographic location. In fact, it is a very old sector, which is located in a mountainous area; it is surrounded by old cracked and unrestored walls with a nearby river and caves which provide favorable resting places for sandflies.

According to the WHO, the urbanization rate is indicated as a key factor in the increase of leishmaniasis [22]. Transmission of leishmaniasis generally occurs in rural areas [21], where it could be linked to human behavior through the presence of animals and the accumulation of their waste near habitats [23]. Thus, Boussaa et al. have confirmed that this factor has a considerable influence on sandfly populations and therefore on the epidemiology of the disease. The abundance of sandflies seems to decrease with the increase in the urbanization rate and certain potential vectors could disappear [24]. In addition, the movement of populations from neighboring rural households to periurban areas can increase the number of leishmaniasis cases due to poor quality of life and socioeconomic status $[25,26]$. These factors constitute favorable conditions for the spread of reservoir hosts and vectors and therefore for the spread of the disease [25].

Regarding the clinical study of leishmaniasis, the age distribution of CL and VL cases showed that no age group was spared from leishmaniasis with a predominance of children aged less than 10 years. This dominance could be explained by the weakness of their immune system and therefore by the inability to defend themselves against infection. In addition, this may also be due to the habits of children who often play near breeding sites, which makes them prone to insect bites [27]. In addition, this study also showed a slight predominance of the male gender with leishmaniasis. This could be explained mainly by the rural character of the provinces where the activities of the population depend closely on the breeding generally practiced by men. The environment in which this type of activity is carried out provides sufficient organic matter favoring the multiplication of sandfly larvae [28]. In addition, this dominance could also be linked to the etiological character of the species $P$. perniciosus which is known to be exophilic [29].

As for environmental factors, leishmaniasis is strongly affected by variations in precipitation, temperature, and humidity. Global warming and soil degradation have a common impact on the epidemiology of leishmaniasis [30]. Indeed, the life cycle of leishmaniasis is sensitive to changes in temperature, rainfall, and humidity. These changes can affect parasites, vectors, and reservoirs by modifying the distribution and influencing survival rates, the population size as well as their dynamic interaction and their territorial expansion [31]. 
Among climatic factors, temperature is the main factor influencing the spread of leishmaniasis. Indeed, even the smallest variations in temperature can have a profound impact on the development cycle of Leishmania promastigotes in sandflies and on their infesting power [32] and thus allow the parasite to be transmitted where the disease was not previously endemic [30]. Another factor that can influence the spread of this parasitosis is the vegetation which also plays an important role in the process of proliferation and growth of sand flies and therefore in the outbreaks of leishmaniasis [33]. The visceral form transmission generally occurs in areas with abundant vegetation [30]. Indeed, Ready reported that deforestation leads to an increase in leishmaniasis [34].

For this, the correlation coefficients between climatic factors and the prevalence of leishmaniasis were calculated. The results obtained showed that humidity, temperature, and precipitation affect the distribution, especially of the visceral form.

On the other hand, a positive correlation was observed between the prevalence of CL and VL and the vicinity with leishmaniasis foci, which remains a major risk responsible for the increase in the number of cases. The provinces having recorded a maximum number of cases are located in the vicinity of the provinces known as foci of leishmaniasis, in particular Taounate and Taza Provinces $[15,19]$.

\section{Conclusions}

Recently, there are a number of challenges for controlling and preventing leishmaniasis. Our results showed that socioeconomic factors, mainly urbanization, contribute significantly to the maintenance of both CL and VL. However, VL is strongly associated with environmental influences. As a result, in this region, control and prevention strategies must be oriented according to each risk factor studied in order to have a more effective result.

\section{Data Availability}

All data generated during the study are included in this article. More details and precision are available from the author via maryam.hakkour@gmail.com upon reasonable request.

\section{Conflicts of Interest}

The authors declare that they have no conflicts of interest.

\section{Acknowledgments}

The authors would like to thank the delegates, animators, and physicians of the provinces studied in this paper for providing all the necessary information on the samples and the study area.

\section{References}

[1] A. Oryan and M. Akbari, "Worldwide risk factors in leishmaniasis," Asian Pacific Journal of Tropical Medicine, vol. 9, no. 10, pp. 925-932, 2016.
[2] WHO, "Control of the leishmaniases: report of a meeting of the who expert commitee on the control of leishmaniases," Tech, Rep, Ser949, WHO, Geneva, Switzerland, 2010.

[3] M. Rhajaoui, "Les leishmanioses humaines au Maroc: une diversité nosogéographique," Pathologie Biologie, vol. 59, no. 4, pp. 226-229, 2011.

[4] M. Roberts, "Current understandings on the immunology of leishmaniasis and recent developments in prevention and treatment," British Medical Bulletin, vol. 75, no. 76, pp. 115-130, 2006.

[5] Ministère de la Santé, "Enquête nationale sur la population et la santé familiale," 2000, https://www.sante.gov.ma/ Publications/Etudes_enquete/Pages/default.aspx.

[6] M. Mniouil, H. Fellah, F. Amarir et al., "Epidemiological characteristics of visceral leishmaniasis in Morocco (1990-2014): an update," Acta Tropica, vol. 170, pp. 169-177, 2016.

[7] Ministère de la Santé, "Enquête nationale sur la population et la santé familiale," 2017, https://www.sante.gov.ma/ Publications/Etudes_enquete/Pages/default.aspx.

[8] PNCL, "Nouveau découpage territorial du royaume," Portail National des Collectivités Territoriales, Casablanca, Morocco, 2015, http://www.pncl.gov.ma/fr/News/Alaune/Pages/Nouveaudécoupage-régional-du-Royaume-.aspx.

[9] HCP, "Recensement général de la population et de l'habitat, haut commissariat au plan," 2014, http://rgph2014.hcp.ma/ downloads/Publications-RGPH-2014_t18649.html.

[10] HCP, Carte de la Pauvreté et de la Vulnérabilité au Maroc, Haut Commissariat au Plan, Casablanca, Morocco, 2007, https:// www.hcp.ma/Indicateurs-provinciaux-de-la-pauvrete-et-de-lavulnerabilite_a648.htm.

[11] HCP, Projections de la Population des Provinces et Prefectures de la Regiontanger-Tetouan-al Hoceima 2014-2030, HautCommissariat au Plan, Casablanca, Morocco, 2018, https:// www.hcp.ma/region-tanger/attachment/995544/.

[12] HCP, Monographie de la région Tanger-Tétouan-Al Hoceima, Haut-Commissariat au Plan, Casablanca, Morocco, 2015.

[13] M. Mukaka, "A guide to appropriate use of Correlation coefficient in medical research," Malawi Medical Journal, vol. 24, no. 3, pp. 69-71, 2012.

[14] J. Rioux, J. Mahjour, M. Gallego et al., "Leishmaniose cutanée humaine à Leishmania infantum Mon-24 au Maroc," Bulletin de la Société Française de Parasitologie, vol. 14, no. 2, pp. 179-183, 1996.

[15] N. Guessous-Idrissi, A. Hamdani, A. Rhalem et al., "Epidemiology of human visceral leishmaniasis in Taounate, a northern province of Morocco," Parasite, vol. 4, no. 2, pp. 181-185, 1997.

[16] N. Guessous-Idrissi, S. Chiheb, A. Hamdani et al., "Cutaneous leishmaniasis: an emerging epidemic focus of Leishmania tropica in north Morocco," Transactions of the Royal Society of Tropical Medicine and Hygiene, vol. 91, no. 6, pp. 660-663, 1997.

[17] H. El Miri, C. Faraj, O. Himmi et al., "Cutaneous leishmaniasis in Ouazzane and Sidi Kacem provinces, Morocco (1997-2012)," Bulletin de la Société de pathologie exotique, vol. 109, no. 5, pp. 376-380, 2016.

[18] WHO, "Report on global surveillance of epidemic-prone infectious diseases-leishmaniasis," WHO, Geneva, Switzerland, 2000, http://www.who.int/csr/resources/publications/ CSR_ISR_2000_1leish/en/.

[19] M. Hakkour, A. Hmamouch, M. M. El Alem et al., "New epidemiological aspects of visceral and cutaneous 
leishmaniasis in Taza, Morocco," Parasites \& Vectors, vol. 9, no. 1, p. 612, 2016.

[20] M. Hakkour, M. Mahmoud El Alem, A. Hmamouch et al., "Leishmaniasis in northern Morocco: predominance of Leishmania infantum compared to Leishmania tropica," BioMed Research International, vol. 2019, p. 14, 2019.

[21] WHO, Leishmaniasis, WHO, Geneva, Switzerland, 2017, http://www.who.int/mediacentre/factsheets/fs375/en/.

[22] WHO, "Urbanization: an increasing risk factor for leishmaniasis," Weekly Epidemiological Record (WER), vol. 77, pp. 365-372, 2002.

[23] W. D. S. Nunes, S. R. Araújo, and C. M. L. Calheiros, "Epidemiological profile of leishmaniasis at a reference service in the state of Alagoas, Brazil, from January 2000 to September 2008," Brazilian Journal of Infectious Diseases, vol. 14, no. 4, pp. 342-345, 2010.

[24] S. Boussaa, B. Pesson, and A. Boumezzough, "Phlebotomine sandflies (diptera: psychodidae) of marrakech city, Morocco," Annals of Tropical Medicine \& Parasitology, vol. 101, no. 8, pp. 715-724, 2010.

[25] R. Reithinger, M. Mohsen, and T. Leslie, "Risk factors for anthroponotic cutaneous leishmaniasis at the household level in Kabul, Afghanistan," PLoS Neglected Tropical Diseases, vol. 4, no. 3, p. e639, 2010.

[26] M. A. Ghatee, I. Sharifi, A. Haghdoost et al., "Spatial correlations of population and ecological factors with distribution of visceral leishmaniasis cases in southwestern Iran," Journal of Vector Borne Diseases, vol. 50, no. 3, pp. 179-87, 2013.

[27] H. Arroub, A. Alaoui, M. Lemrani, and K. Habbarin, " $\mathrm{Cu}-$ taneous leishmaniasis in foumjamâa (Azilal, Morocco): micro-environmental and socio-economical risk factors," Journal of Agriculture, Forestry and the Social Sciences, vol. 8, pp. 10-16, 2012.

[28] K. Lahouiti, A. E. O. Alami, A. Hmamouch, and K. Bekhti, "Phototropism of sand flies species (diptera: psychodidae) collected in a rural locality in Central Morocco," Journal of Parasitology and Vector Biology, vol. 6, no. 5, pp. 66-74, 2014.

[29] IRMA, Information Resources Management Association, Public Health and Welfare: Concepts, Methodologies, Tools, and Applications: Concepts, Methodologies, Tools, and Applications, IGI Global, Pennsylvania, PA, USA, 2017.

[30] WHO, Leishmaniasis, Epidemiological Situation, WHO, Geneva, Switzerland, 2019, https:/www.who.int/leishmaniasis/ burden/en/.

[31] L. Bounoua, K. Kahime, L. Houti et al., "Linking climate to incidence of zoonotic cutaneous leishmaniasis (L. major) in pre-Saharan North Africa," International Journal of Environmental Research and Public Health, vol. 10, no. 8, pp. 3172-3191, 2013.

[32] J. A. Rioux, J. P. Aboulker, G. Lanotte, R. Killick-Kendrick, and A. Martini-Dumas, "Écologie des leishmanioses dans le sud de la France," Annales de Parasitologie Humaine et Comparée, vol. 60, no. 3, pp. 221-229, 1985.

[33] A. Oryan and M. Akbari, "Worldwide risk factors in leishmaniasis," Asian Pacific Journal of Tropical Medicine, vol. 9, no. 10, pp. 925-932, 2016.

[34] P. D. Ready, "La aparición de leishmaniasis y el cambio climático," Revue Scientifique et Technique de l'OIE, vol. 27, no. 2, pp. 399-412, 2008. 